Meta

Journal des traducteurs

Translators' Journal

\title{
Les flux d'information dans les réunions interlinguistiques et l'interprétation de conférence : premières observations
}

\section{Daniel Gile}

Volume 34, numéro 4, décembre 1989

URI : https://id.erudit.org/iderudit/002900ar

DOI : https://doi.org/10.7202/002900ar

Aller au sommaire du numéro

Éditeur(s)

Les Presses de l'Université de Montréal

ISSN

0026-0452 (imprimé)

1492-1421 (numérique)

Découvrir la revue

Citer cet article

Gile, D. (1989). Les flux d'information dans les réunions interlinguistiques et l'interprétation de conférence : premières observations. Meta, 34(4), 649-660. https://doi.org/10.7202/002900ar d'utilisation que vous pouvez consulter en ligne. 


\title{
LES FLUX D'INFORMATION DANS LES RÉUNIONS INTERLINGUISTIQUES ET L'INTERPRÉTATION DE CONFÉRENCE : PREMIËRES OBSERVATIONS
}

\author{
DANIEL GILE \\ CEEI (ISIT) et INALCO, Paris, France
}

\section{INTRODUCTION}

Jusqu'ici, les travaux de réflexion et de recherche sur l'interprétation de conférence étaient fondés sur le point de vue de l'interprète, son rôle tel que perçu par lui-même, ses aptitudes, sa formation, ses limites personnelles.

D'après le point de vue officiel de l'ÉSIT (École supérieure d'interprètes et de traducteurs de l'université Paris III), qui prévaut actuellement dans la profession, les interprètes ont pour mission de restituer fidèlement et intégralement le message véhiculé par chaque intervention.

Si l'on devait appliquer rigoureusement cette définition sur le terrain, force serait de constater que les interprètes sont en général incapables de restituer ce message dans tous les détails dès qu'interviennent certaines difficultés auxquelles on n'échappe que rarement: intervention dense, rapide, lue, noms propres, termes techniques inconnus de l'interprète, chiffres, énumérations, etc. (voir Gile : 1984a,b).

Cette constatation, que fait inévitablement le jeune interprète dès ses premiers pas sur le terrain, est source de frustration, puis parfois d'un certain laisser-aller si les obstacles se révèlent «insurmontables».

Néanmoins, face aux interprètes découragés qui ont l'impression de mal faire leur travail, les réactions des délégués sont souvent étonnantes par leur générosité apparente. De toute évidence, les critères ne sont pas les mêmes des deux côtés du microphone (voir Gile: 1983 ).

En réalité, les interprètes, qui tendent à considérer qu'ils travaillent dans un univers interpréto-centrique fermé, sont au service des délégués dans un environnement de communication centré autour de ces derniers. Cet environnement est en mutation constante, et a beaucoup évolué depuis les années 50 et 60 au cours desquelles furent écrits les premiers livres sur l'interprétation (notamment le Manuel de l'interprète de Jean Herbert: 1952 et l'Interprète dans les conférences internationales de Danica Seleskovitch: 1968). Aujourd'hui, les conférences sont plus nombreuses, plus variées, plus techniques; actuellement, le secteur privé occupe une place plus importante du marché que les organisations internationales et ministères, et les interprètes ne sont plus des «oiseaux rares», mais des professionnels qui inspirent beaucoup moins la curiosité et l'admiration et dont on attend une compétence professionnelle précise.

Dans ces conditions, nous pensons que la réflexion sur l'interprétation doit évoluer elle aussi. Dans un environnement devenu véritablement professionnel (certains parlent d'un environnement «fonctionnarisé»), il ne s'agit plus de dévoiler le mystère de la «simultanéité» de l'écoute et de l'interprétation, ni de redécouvrir que la traduction ne consiste pas à transcoder, mais à assimiler un message et à le réexprimer; ces principes 
fondamentaux, abondamment commentés et illustrés par D. Seleskovitch, K. Déjean-leFéal et $M$. Lederer, méritent toujours d'être rappelés en raison de leur importance capitale, certes, mais le temps est venu d'aller plus loin, d'étudier de manière plus approfondie les mécanismes mentaux de l'interprétation (voir par exemple Moser: 1978 et Gile: 1985a), et de se tourner vers l'environnement extérieur et ses contraintes agissant sur l'interprétation (voir Déjean-le-Féal: 1978, Quicheron: 1984 et Gile : 1984a,b).

En tout état de cause, il ne faut plus se cantonner dans un nombrilisme interprétocentrique, mais se tourner également vers les délégués, leur environnement et leurs besoins. Nous avons entrepris récemment une observation systématique de cette autre face de l'univers des conférences internationales - la plus importante - telle que vue à travers l'ceil des délégués, et recueilli quelques centaines de commentaires. À ce stade, l'analyse est nécessairement très incomplète. Quelques convergences importantes se sont toutefois d'ores et déjà dégagées et font apparaître une certaine image. Le présent article en trace les grandes lignes, en commençant par la caractérisation informationnelle des principaux types de réunions interlinguistiques auxquelles travaillent les interprètes de conférence, et en généralisant par la suite. Nous espérons que cette image pourra être corrigée, affinée et complétée par des collègues interprètes, des organisateurs de conférences internationales, des délégués participant à de telles réunions et des chercheurs spécialisés dans la communication qui voudront bien apporter leur contribution sous forme de critiques et de commentaires.

\section{LES RÉUNIONS INTERLINGUISTIQUES: QUELQUES EXEMPLES}

Les réunions interlinguistiques auxquelles travaillent les interprètes de conférence sont très variées en ce qui concerne leurs thèmes, l'identité des participants et les modalités d'organisation. Toutefois, au regard de la transmission informationnelle qui y intervient, elles peuvent être classées en un petit nombre de catégories. Dans les pages suivantes, nous présentons quelques types de réunions interlinguistiques qui couvrent une grande partie de l'activité professionnelle de l'interprète de conférence. En réalité, ces catégories ne sont pas toujours aussi nettement tranchées, et il arrive qu'elles s'interpénètrent. Il existe par ailleurs d'autres réunions qu'il est difficile de classer dans ces catégories. Toutefois, les paramètres fondamentaux que l'on peut dégager sur la base de l'étude des cas-types présentés ci-dessous nous semblent avoir une validité générale qui couvre l'ensemble des réunions interlinguistiques auxquelles travaillent les interprètes de conférence.

\section{LES GRANDES CONFÉRENCES SCIENTIFIQUES ET TECHNIQUES}

Les grandes conférences scientifiques et techniques durent plusieurs jours, parfois une semaine ou plus, et rassemblent en général plusieurs centaines de participants. Elles se composent de communications, généralement présentées dans le cadre de «sessions» d'une demi-journée ou d'un quart de journée, de «séances de posters» et de démonstrations de matériel. Les communications, préparées avant la réunion, sont présentées en un quart d'heure à une demi-heure dans la plupart des cas, et leur présentation est suivie de brèves périodes de questions et de réponses et parfois de «tables rondes».

L'information présentée à ces conférences est abondante et dense; il en résulte que même pour des participants dotés d'un pouvoir de concentration et d'assimilation très supérieur à la moyenne, elle ne peut être appréhendée dans sa totalité en séance (notamment en ce qui concerne les formules et démonstrations mathématiques, les descriptions de matériel, les tableaux statistiques), mais seulement dans ses grandes lignes ou dans des points présentant un intérêt particulier pour l'auditeur. 
En outre, les communications comportent fréquemment des éléments spécialisés qui débordent les centres d'intérêt et les domaines véritablement maîtrisés par les auditeurs. À titre d'exemple, au cours du Colloque international d'intelligence artificielle qui s'est tenu à Marseille du 24 au 27 octobre 1984, ont été abordées dans quelque détail des questions géologiques, médicales, linguistiques, nucléaires, aéronautiques, mathématiques et autres. En toute probabilité, aucun des délégués n'était véritablement compétent dans tous ces domaines à la fois. Cette diversité dans les spécialités constitue la règle plutôt que l'exception, puisqu'elle se retrouve dans tous les actes des grandes conférences scientifiques que nous avons recueillis depuis six ans, soit plusieurs dizaines de tomes. En conséquence, indépendamment de la densité et de la technicité de l'information présentée, il existe inévitablement des auditeurs pour qui les communications restent partiellement obscures, voire complètement hermétiques, comme nous l'ont confirmé la quasi-totalité des délégués que nous avons pu interroger à ce sujet.

De ces observations, il résulte qu'indépendamment de l'intervention de l'interprète et de toute considération linguistique, la perte d'information à l'écoute chez les auditeurs est considérable dans ce type de réunion. En fait, la plupart des délégués que nous avons interrogés jusqu'ici confirment qu'en se rendant aux séances de travail des grandes conférences scientifiques et techniques, ils ne s'attendent pas à acquérir un bagage informationnel important, mais plutôt à saisir des idées générales, quitte à rechercher un complément éventuel hors-séance, voire hors-conférence, le plus souvent par la voie écrite (lecture attentive des Actes du colloque, échange de lettres, de publications, etc.).

Sur le plan linguistique, il n'est pas rare que les participants aux grandes conférences scientifiques et techniques comprennent peu ou prou une partie des langues d'intervention étrangères sous leur forme de langues de spécialité écrites, et notamment les termes spécialisés relevant de leur discipline, surtout quand il s'agit de langues dominantes (actuellement, l'anglais dans de nombreuses disciplines). Par contre, ils sont souvent incapables de s'exprimer dans de bonnes conditions dans ces langues étrangères et ont parfois des problèmes de compréhension de l'oral; la présence des interprètes à ces conférences résulte essentiellement de ces problèmes.

\section{LES «SÉMINAIRES» ET COURS TECHNIQUES}

Certains cours et stages de formation techniques sont organisés avec la participation d'intervenants étrangers, ou avec des stagiaires de différentes langues. C'est notamment le cas des «séminaires» que tiennent en France plusieurs organismes de formation qui recrutent des conférenciers étrangers, le plus souvent américains. Les stagiaires, quelques dizaines en général, présentent une homogénéité plus grande que les participants aux grandes conférences scientifiques et techniques, en ce sens qu'ils sont tous concernés par le thème de la réunion, mais leurs connaissances techniques en la matière sont parfois assez inégales.

Ces cours et «séminaires» sont des réunions techniques où le flux est essentiellement unidirectionnel (du conférencier vers les stagiaires), bien que des périodes de questions et réponses y soient prévues. L'information est spécialisée, dense et souvent quantitativement importante, mais sa présentation est structurée de manière à faciliter son assimilation, à la différence de l'information présentée dans les grandes conférences scientifiques et techniques. Néanmoins, un support écrit est prévu pour faciliter la transmission de l'information, qui ne peut en général être entièrement assimilée en séance.

Ces cours et «séminaires» techniques sont proches, sur le plan de la transmission informationnelle, des présentations de produits par des constructeurs et fournisseurs. Néanmoins, dans ces présentations, la différence cognitive entre les orateurs et les 
auditeurs est moins marquée, dans la mesure où la spécialisation de l'ensemble des participants par rapport à l'information échangée est plus grande.

\section{LES RÉUNIONS DE TRAVAIL DANS LES ORGANISATIONS INTERNATIONALES}

Les organisations internationales (ONU, UNESCO, UNICEF, OCDE, CCE, Conseil de l'Europe, Parlement européen, BIRD, OACI, ESA, OMS, etc.) accomplissent une partie importante de leur travail à travers l'activité de comités et groupes de travail qui se réunissent régulièrement dans le cadre de l'exécution d'un mandat qui leur est confié. Ces réunions peuvent durer quelques dizaines de minutes ou plusieurs jours, et se déroulent selon un ordre du jour qui précise les points qui devront être abordés.

En général, il y a dans ces réunions non pas présentation unilatérale de communications orales, comme c'est le cas dans les grandes conférences scientifiques et techniques, mais discussion sur un ensemble de points, l'information de base étant le plus souvent présentée dans des documents de travail préparés à l'avance par le secrétariat du groupe et envoyés aux participants.

L'essentiel de l'information ayant été présenté par écrit et assimilé préalablement à la réunion, le flux informationnel en séance de travail est moins important et moins dense, et se compose de questions, de commentaires et d'éclaircissements plutôt que d'informations nouvelles.

Les participants, nettement moins nombreux que dans les grandes conférences scientifiques et techniques (leur nombre varie dans la plupart des cas entre trois ou quatre et quelques dizaines), se connaissent bien pour la plupart et ont généralement l'occasion de se voir dans le cadre d'autres réunions de travail. Nombreux sont les polyglottes parmi eux, et ils peuvent communiquer directement tant bien que mal hors séance; toutefois, la contribution des interprètes leur permet d'une part de s'exprimer avec toute leur éloquence dans leur langue maternelle (ou, à défaut, dans la langue de la conférence qu'ils maîtrisent le mieux), et d'autre part de suivre les échanges et d'y participer à un rythme soutenu en cours de réunion.

Sur le plan de la communication, les réunions de travail dans les organisations internationales diffèrent donc sensiblement des grandes conférences scientifiques et techniques.

- La quantité d'information qui y est transmise est bien plus faible, et le flux informationnel est écrit et en grande partie préalable à la séance de travail plutôt que oral et concomitant à la réunion.

- L'information concerne l'ensemble des délégués; cognitivement, elle est accessible à tous.

- Les flux d'information liés à la réunion sont continus et se situent essentiellement hors-séance, par opposition au flux chronologiquement ponctuel des grandes conférences scientifiques et techniques.

- Les flux ont essentiellement la forme d'échanges, les participants réagissant aux différentes interventions, par opposition aux grandes conférences scientifiques et techniques où il y a présentation d'information par les intervenants, mais sans véritable échange.

\section{LES NÉGOCLATIONS}

Dans les négociations (politiques, économiques, commerciales, etc.), le nombre de destinataires est plus limité que dans les trois types de réunions évoqués plus haut, car les interlocuteurs sont des délégations, représentées par leurs chefs, plutôt que des individus. Par ailleurs, les flux n'y ont pas une finalité d'information, comme dans les conférences scientifiques et techniques et les cours et «séminaires», mais un but pratique, puisqu'il s'agit d'arriver à un accord concret entre les parties en présence. 
Les interlocuteurs connaissent bien leurs positions respectives et leurs dossiers, et l'information échangée dans ce type de réunion est peu abondante, mais en général précise, notamment en ce qui concerne les chiffres et les noms propres. Dans leur forme, les échanges se caractérisent par leur nature discontinue (interruptions de séance pour consultations), par une activité rédactionnelle importante (propositions, contre-propositions, accord écrit) et par un style à tendance plutôt formelle et juridique.

\section{LES VISITES MINISTÉRIELLES}

Les interprètes sont souvent amenés à travailler pour des ministres à l'occasion des visites de leurs homologues étrangers. L'ordre de grandeur de la durée d'une telle réunion est d'une heure, et les participants y sont peu nombreux; les ministres en question, parfois des chefs de cabinet, des conseillers, des spécialistes, des ambassadeurs, d'autres personnes concernées. Ces visites ont en général un caractère politique, et quand un point technique y est abordé, c'est le plus souvent d'une manière globale, les détails précis étant traités au niveau des spécialistes dans les ministères et autres organismes concernés.

L'information échangée au cours de ces visites est donc quantitativement peu importante, peu dense et peu spécialisée. Les paroles des interlocuteurs sont toutefois souvent chargées d'éléments politiques importants.

\section{LES DÉBATS PARLEMENTAIRES}

Dans certains pays plurilingues, les débats parlementaires sont systématiquement interprétés pour des raisons constitutionnelles, bien que tous les participants comprennent les langues concernées. Dans ces débats, les échanges sont moins informatifs (les parlementaires se connaissent et connaissent les dossiers évoqués) que politiques, souvent polémiques, et se distinguent dans leur forme par d'importants éléments juridiques et de procédure.

\section{LES DÉBATS RADIO- OU TÉLÉDIFFUSÉS}

Différents programmes à la radio et à la télévision comportent des débats avec la participation de personnalités étrangères qui ne maîtrisent pas la langue de l'émission. Les interprètes sont appelés à intervenir dans ces débats.

Dans ce type de réunion, les échanges portent davantage sur des opinions que sur des informations, et sont destinés à alimenter une conversation intéressante pour les auditeurs ou les télespectateurs autant, sinon plus, que pour informer. L'information est donc peu dense, peu abondante et peu spécialisée; elle est souvent sollicitée par des questions et a fréquemment une nature anecdotique. Le registre linguistique employé est moins officiel que dans les autres types de réunions cités plus haut.

\section{LES CONFÉRENCES DE PRESSE}

Les flux des conférences de presse sont des flux «en éventail», une personnalité ou un organisme (société, association, institution) se trouvant face à face avec un groupe de journalistes. Sur le plan formel, ils peuvent être essentiellement unidirectionnels (quand il s'agit d'une conférence de presse organisée par des fabricants ou constructeurs à l'occasion du lancement d'un nouveau produit par exemple), auquel cas ils s'accompagnent souvent d'une documentation écrite abondante distribuée aux journalistes, ou bidirectionnels, quand il s'agit d'une conférence de presse accordée par une personnalité politique qui accepte de répondre aux questions des journalistes par exemple; dans ce deuxième cas, la documentation écrite est généralement absente ou quantitativement restreinte (elle se limite souvent à un communiqué de presse).

Dans ce type de réunion, le flux informationnel proprement dit est unidirectionnel et va toujours de la personnalité vers les journalistes et l'information transmise est en général peu abondante, bien qu'elle puisse parfois être très spécialisée. 
Dans tous les cas, elle est accessible à l'ensemble des interlocuteurs, contrairement à l'information émise lors des grandes conférences scientifiques et techniques; d'autre part, contrairement aux participants à ces conférences scientifiques et techniques, les participants aux conférences de presse, tant les journalistes que les personnalités qui leur font face, écoutent en général les échanges avec une attention assez soutenue.

\section{LES CONFÉRENCES DE PERSONNALITÉS INVITÉES}

Il arrive que les interprètes soient amenés à interpréter un discours fait par une personnalité célèbre (ancien chef d'État, premier ministre, etc.) invitée par l'organisateur et à l'occasion d'un «déjeuner-débat» ou dans le cadre d'une autre réunion.

Le flux informationnel qui caractérise ces réunions est essentiellement unidirectionnel, bien que des questions et réponses suivent souvent l'exposé. Par ailleurs, l'information émise par la personnalité en question est quantitativement faible, généralement peu spécialisée, ne s'accompagne d'aucune documentation écrite et n'est écoutée que d'une oreille modérément attentive, la valeur de l'intervention étant fonction du prestige, de la personnalité bien plus que de son contenu informationnel, dont l'intérêt pour les participants est souvent plutôt modéré.

\section{LES DÎNERS OFFICIELS}

Les dîners officiels peuvent constituer une réunion interlinguistique à part entière ou être donnés dans le cadre d'un autre type de réunion (conférence scientifique, visite ministérielle, etc.). Les interprètes y accomplissent deux fonctions:

a) L'interprétation des propos échangés entre des convives voisins (souvent, dans les dîners officiels des ministères, ce sont les «petites chaises»: les interprètes sont assis derrière les personnalités auxquelles ils sont affectés et interviennent en chuchotant). Cette forme d'interprétation est plus proche de l'interprétariat «de liaison» que de la véritable interprétation de conférence, et ne sera pas traitée ici.

b) L'interprétation des interventions officielles, dites «discours de table». Il s'agit des discours des personnalités officielles, faits avant, pendant ou après le dîner. Leur contenu informationnel est généralement faible (il arrive toutefois qu'ils comportent des éléments politiques importants), avec de nombreux clichés répétitifs (salutations, remerciements, compliments, félicitations, voeux de réussite, etc.). Dans leur forme, ils se caractérisent par un registre plutôt officiel, souvent oratoire, et fréquemment par de nombreuses citations et un ton humoristique.

\section{LES FLUX INFORMATIONNELS DANS LES CONFÉRENCES INTERLINGUISTIQUES : PRINCIPAUX PARAMÈTRES}

Comme il est dit plus haut, les réunions interlinguistiques évoquées schématiquement ci-dessus sous l'angle des flux informationnels ne sont que des archétypes dont il arrive que la réalité s'écarte sensiblement. Néanmoins, dans toutes les variantes et combinaisons de ces archétypes, on retrouve les mêmes paramètres des flux informationnels qui servent non seulement à caractériser les réunions, mais aussi à expliquer les difficultés particulières qui se posent aux interprètes dans chaque type de réunion.

\section{LES HANDICAPS DE L'INTERPRÈTE}

L'interprétation de conférence, et notamment l'interprétation simultanée, est une activité intellectuelle intense qui implique la conduite de trois efforts - effort d'écoute et d'analyse, effort de mémoire à court terme ou de prise de notes, et effort de production du discours - ainsi qu'un délicat réglage du partage de l'attention entre ces efforts (voir Gile: 1985a). Elle comporte donc des difficultés techniques propres aux mécanismes 
mentaux en jeu, difficultés que renforcent certains éléments survenant ponctuellement dans les discours interprétés: les noms propres, les termes techniques, les énumérations, les discours denses et rapides, etc., intensifient les trois efforts et en déréglent l'équilibre (voir Gile: 1984a).

En réalité, les difficultés de la transmission informationnelle en interprétation de conférence sont déterminées par trois éléments : la compétence linguistique de l'interprète, son aptitude intellectuelle en termes de réglage de l'équilibre entre les trois efforts de l'interprétation, et l'écart entre son bagage cognitif et terminologique et celui des autres participants.

En effet, à capacité intellectuelle égale, plus l'interprète est familiarisé avec le thème de la conférence, les concepts, les modes de raisonnement, les noms propres et les termes techniques employés, moins il a d'efforts à fournir pour comprendre le message, moins il charge sa mémoire d'éléments cognitifs nouveaux, et plus la réexpression du message dans la terminologie appropriée est spontanée.

C'est essentiellement à la lumière de ce troisième élément, qui est fonction des caractéristiques informationnelles de la réunion plutôt que des aptitudes personnelles de l'interprète, que nous analysons dans les pages suivantes la situation de celui-ci dans les différents types de conférences interlinguistiques évoqués plus haut.

\section{PARAMÈTRES CARACTÉRISANT L'INFORMATION}

De l'analyse des handicaps de l'interprète présentée ci-dessus, il ressort que la difficulté d'une réunion interlinguistique est notamment fonction de sa TECHNICITÉ, puisque celle-ci accroît l'écart entre le bagage cognitif des délégués et celui de l'interprète. En fait, la seule technicité de la réunion ne constitue pas nécessairement un facteur de difficulté important: il nous est arrivé de travailler à une conférence très spécialisée dont la mission consistait à classer des substances chimiques dans un certain nombre de catégories; le raisonnement des participants était très simple et les noms des substances en question constituaient la seule difficulté terminologique, d'ailleurs fortement atténuée grâce aux listes qui avaient été distribuées aux interprètes comme aux participants.

Quand l'information échangée dans une conférence est quantitativement faible, l'interprète arrive assez vite à circonscrire tant les raisonnements que les concepts de base (voir Gile: 1985b). Ce qui donne à la technicité de l'information un caractère de facteur de difficulté, c'est l'élément QUANTITATIF : quand l'information est technique et quantitativement importante, l'interprète ne dispose pas du temps nécessaire à l'assimilation des raisonnements, des concepts et des termes, même s'il dispose d'une documentation abondante (la lecture et l'exploitation des centaines, voire des milliers de pages de communications, actes, comptes rendus et autres documents qui accompagnent les conférences scientifiques et techniques demandent des dizaines $d$ 'heures que l'interprète ne peut consacrer à ce travail). De même, la seule quantité d'information échangée au cours d'une conférence ne constitue pas un facteur de difficulté, car elle n'est pas nécessairement extérieure au bagage cognitif de l'interprète et ne l'oblige pas nécessairement à intensifier ses efforts d'écoute et d'analyse, de mémoire à court terme et de production du discours. Par contre, conjuguée à un certain degré de technicité, elle se transforme en un obstacle considérable.

\section{PARAMÈTRES CARACTÉRISANT LES FLUX INFORMATIONNELS}

\section{a) Flux réactifs et flux non-réactifs}

Le terme «conférence internationale» tend à évoquer spontanément 1'image d'un forum où des interlocuteurs s'adressent la parole et se répondent en un véritable échange. En réalité, comme il est montré plus haut, dans différents types de réunions 
interlinguistiques (grandes conférences scientifiques et techniques, séminaires et cours techniques, dîners officiels), les échanges sont fort limités.

Pour l'interprète, les réunions à flux réactifs (réunions des groupes de travail dans les organisations internationales, visites ministérielles, négociations, débats) sont moins difficiles. En effet:

- les échanges élargissent le contexte que l'interprète peut analyser et lui facilitent l'assimilation des concepts et des raisonnements de base;

- ils lui donnent aussi l'occasion d'entendre les délégués employer les termes spécialisés plus souvent, dans différents contextes et souvent dans les différentes langues de la réunion, ce qui lui permet de consolider son bagage terminologique.

Il convient de noter toutefois que dans certaines réunions non-réactives, la présentation de l'information sous une forme structurée dans une optique pédagogique (voir la section sur les «séminaires» et «cours techniques» plus haut) lui facilite grandement l'assimilation des concepts et du raisonnement, et lui donne une base cognitive plus solide que celle qu'il se constitue en recueillant et en rassemblant des fragments d'information dans les réunions à flux réactifs.

\section{b) Caractéristiques chronologiques des flux}

Dans les dix archétypes de réunions interlinguistiques présentés ici, on peut distinguer trois catégories de chronologies de flux, que nous désignerons par les termes «flux pendant», «flux pendant-après» et «flux avant-pendant-après» :

(1) Les «flux pendant» ont un cycle de vie qui coïncide avec celui de la réunion : ils prennent naissance dès les premières interventions et se terminent en même temps que les dernières. Les «flux pendant» caractérisent notamment les débats radio- et télédiffusés et les discours de personnalités invitées.

(2) Les «flux pendant-après» commencent au début de la réunion mais se poursuivent audelà de celle-ci : les conférences scientifiques et techniques et les cours et séminaires techniques, où l'exploitation du support écrit et les échanges post-séance complètent le transfert partiel d'information qui intervient à l'écoute des exposés oraux en sont deux exemples typiques.

(3) Les «flux avant-pendant-après» correspondent à des situations où il existe des échanges oraux ou épistolaires entre deux ou plusieurs parties et où des réunions sont organisées pour rassembler l'ensemble des interlocuteurs en un même lieu physique et au même moment pour des échanges plus concentrés, des mises au point et la prise de décisions. Ce genre de flux caractérise notamment les activités des groupes de travail constitués dans les organisations internationales.

Dans les réunions de faible technicité, l'écart entre le bagage cognitif spécifique (relevant de la conférence en question) de l'interprète et celui des délégués est plus important dans les «flux avant-pendant-après» que dans les «flux pendant» et les «flux pendant-après», car dans les réunions où les échanges en séance ne représentent qu'un segment chronologique du flux informationnel, au moment où intervient l'interprète, des informations ont déjà été échangées entre les délégués, alors que dans les deux autres types de réunions, l'interprète prend connaissance des différents éléments d'information en même temps que les participants. D'un autre côté, dans les réunions de type «avantpendant-après», on trouve souvent une documentation abondante sous forme de comptes rendus et de documents de travail qui permettent à l'interprète de renforcer son bagage cognitif spécifique et de réduire ainsi son infériorité cognitive par rapport aux autres participants. Dans les réunions à flux denses et spécialisés, l'influence de ces paramètres chronologiques s'atténue grandement. 


\section{L'ÉQUILIBRE LINGUISTIQUE}

L'équilibre quantitatif entre les différentes langues de la réunion a une importance non négligeable pour les interprètes. En effet, dans une réunion linguistiquement équilibrée (où chacune des langues de la conférence est employée aussi souvent que les autres), les interprètes ont davantage l'occasion d'entendre les délégués employer les termes appropriés dans les différentes langues; en outre, ils y ont davantage de chances de trouver une documentation multilingue. Ces deux facteurs constituent une aide précieuse à l'acquisition du bagage terminologique nécessaire.

Par ailleurs, dans chaque réunion interlinguistique, on peut distinguer, sociolinguistiquement parlant, un groupe de participants qui font appel à l'interprétation (pour comprendre ou pour s'exprimer) et un groupe de participants qui n'utilisent pas les services des interprètes. Quand le premier groupe est relativement important, tant les intervenants que les organisateurs prennent conscience des problèmes linguistiques et accordent davantage d'attention à l'interprétation: le président de séance demande aux intervenants de parler lentement et clairement, les orateurs eux-mêmes viennent parfois trouver les interprètes en cabine pour leur donner une copie de leur texte ou pour leur expliquer les principales idées et les mots clés de leurs interventions respectives, et il arrive que l'on organise une séance de «briefing» à l'intention des interprètes avant la réunion. Quand les interprètes ont un petit nombre de «clients» dans la salle, par contre, les participants perdent de vue les problèmes linguistiques et d'interprétation, ont tendance à s'exprimer sans égards pour leurs collègues étrangers, et vont jusqu'à ne plus se servir des microphones, ce qui rend l'interprétation très difficile, voire impossible.

\section{PARAMÈTRES ORGANISATIONNELS}

Sous l'angle des flux informationnels, l'élément organisationnel le plus important pour les interprètes dans les réunions interlinguistiques est indubitablement la documentation, car c'est la documentation qui leur permet de combler de la manière la plus efficace une partie de leurs lacunes cognitives et terminologiques.

À propos de ces documents, on évoquera en premier lieu le moment de leur préparation et de leur diffusion. En effet, les documents préparés et distribués aux interprètes plusieurs semaines avant la réunion leur permettent de faire des recherches en bibliothèque pour renforcer leur connaissance du sujet et enrichir leur lexique spécialisé. Par contre, les documents qui leur sont remis en séance, parfois quelques minutes avant l'intervention concernée, ne peuvent qu'être lus en diagonale et préparés d'une manière approximative avec les moyens du bord, c'est-à-dire à l'aide d'éventuels lexiques et dictionnaires emportés en cabine, le plus souvent insuffisants. Une telle préparation reste nécessairement superficielle et incomplète, même sur le plan terminologique.

Par ailleurs, certains documents constituent des «enveloppes cognitives complètes»: c'est le cas des Actes dans les conférences scientifiques et techniques et des documents de travail des comités et groupes de travail constitués au sein des organisations internationales. D'autres servent de support à une présentation: dans les cours techniques et "séminaires», les documents fournis aux participants et interprètes sont le plus souvent des copies sur papier des transparents utilisés par le conférencier et contiennent des schémas et énumérations de points que le conférencier commente, plutôt qu'un véritable texte. D'autres encore ont une fonction administrative: ordre du jour ou programme des conférences et communications, renseignements pratiques à l'intention des participants. À l'évidence, l'utilité de ces différents types de documents pour les interprètes est inégale.

Enfin, la composition linguistique des documents est importante sur le plan terminologique: plus on se rapproche de l'équilibre linguistique, plus les interprètes sont susceptibles d'y trouver les «équivalences terminologiques» spécifiques dont ils ont besoin pour la réunion. 
D'autres aspects de l'organisation de la réunion ont une influence considérable sur la situation des interprètes. L'existence d'un ordre du jour ou d'un programme précisément définis et respectés en séance leur permet d'élaborer leur propre programme de travail, tant avant la conférence qu'en cours de réunion (répartition du travail en fonction des préférences et spécialités éventuelles de chaque membre de l'équipe, préparation des interventions à l'aide des textes écrits); les incertitudes et changements improvisés dans les programmes mettent les efforts des interprètes en échec en les empêchant d'organiser leur préparation de cette manière.

Le déroulement chronologique de la réunion a lui aussi une importance cruciale, car la fatigue peut entraîner une baisse sensible de la qualité de l'interprétation si les sessions sont trop longues (plus de trois heures à trois heures et demie), s'il n'y a pas de «pauses-café» et si les «pauses-déjeuner» sont trop courtes et ne permettent pas aux interprètes de se reposer.

On peut également évoquer d'autres éléments organisationnels qui ont un retentissement certain sur l'interprétation: l'emplacement de la cabine d'interprétation par rapport aux intervenants et à l'écran sur lequel sont projetés les transparents et les diapositives (conditions de visibilité), les conditions acoustiques et électroniques (qualité du son), la taille de la cabine, l'éclairage et l'aération dans la salle et la cabine (conditions de confort), ainsi que d'autres facteurs techniques qui ne sont pas fonction des types de flux informationnels intervenant dans les réunions concernées.

\section{PARAMĖTRES PSYCHOLOGIQUES ET SOCIOLOGIQUES}

Outre les paramètres «objectifs» évoqués dans les pages précédentes, des paramètres «subjectifs» de nature psychologique et sociologique ont une influence considérable sur les flux effectifs d'information dans les conférences interlinguistiques.

En effet, comme le laisse entrevoir la présentation des dix archétypes de réunions au début du présent exposé, l'incorporation d'éléments d'information dans le discours des intervenants n'implique pas leur assimilation par les autres participants, et ce pour des raisons cognitives (c'est le cas des communications spécialisées dans les grandes conférences scientifiques et techniques qui ne sont pas à la portée de tous les participants), mais aussi pour des raisons motivationnelles: une partie plus ou moins importante des informations émises lors des réunions interlinguistiques n'intéresse pas tout l'auditoire, comme le montrent notamment les feuilles d'évaluation que les organisateurs de diverses manifestations de ce type demandent aux participants de remplir.

En réalité, les motivations des délégués aux différentes réunions interlinguistiques sont variables, tant quantitativement que qualitativement. La grande majorité des délégués que nous avons interrogés jusqu'ici (le résultat vaut pour toutes les catégories de réunions) affirment que la recherche d'informations en séance ne constitue pas la raison principale de leur participation à la réunion; ils jugent en effet souvent inintéressantes, parce que banales, redondantes ou mal présentées, les informations émises en séance. Dans leurs réponses, ils évoquent plutôt les prises de contact et les renouvellements de contacts avec des confrères, d'éventuels échanges d'informations hors-séance, l'occasion de prendre la parole soi-même, le tourisme (!) et d'autres motivations totalement étrangères à l'échange d'informations.

Ces facteurs humains ne facilitent pas le travail des interprètes. En effet, quand ils se désintéressent de l'information émise en réunion, les participants se désintéressent également des problèmes linguistiques et d'interprétation. Dans ces conditions, les récriminations des interprètes qui se trouvent face à un orateur lisant très vite un texte qui ne leur a pas été fourni, ou à une assistance qui néglige l'emploi du microphone au moment des questions et réponses, sont totalement inefficaces. Bien plus, elles agacent les participants 
qui peuvent se sentir agressés par ce tiers qu'ils considèrent le plus souvent comme étranger à leur groupe.

\section{EN GUISE DE CONCLUSION : QUESTIONS D'INTERFAÇAGE}

Des observations faites dans les pages précédentes, il ressort clairement que les conditions de communications dans les conférences internationales sont très variées, et qu'elles affectent non seulement la capacité des interprètes de restituer intégralement les messages des intervenants, mais aussi la nature même de leur rôle aux yeux des participants.

La situation est bien connue des interprètes, qui la vivent quotidiennement, et qui s'y adaptent tant bien que mal. Nous pensons toutefois qu'un travail de réflexion supplémentaire, notamment de la part des responsables des écoles d'interprètes, pourrait être utile. En effet, une sensibilisation en cours de formation est susceptible de rendre moins longue et moins pénible la période d'adaptation des jeunes diplômés dont un certain nombre passe de la surprise à l'irritation, de l'irritation aux doutes, et des doutes au découragement et parfois au laisser-aller pendant les premiers mois, ou les premières années de leur vie professionnelle en réalisant l'écart entre l'univers interpréto-centrique dans lequel ils ont été formés et la réalité des conférences internationales. Peut-être pourrait-on envisager également de mieux préparer les élèves-interprètes à des exigences autres que la restitution intégrale d'un message dans les conditions théoriques idéales de la consécutive ou de la simultanée, et ce tant par une orientation pédagogique que par des exercices appropriés, aussi bien sur le plan de l'acte interprétatif que sur le plan du comportement social.

Du côté des organisateurs des conférences internationales - dont beaucoup, sur le marché privé, n'en savent pas assez sur l'interprétation pour comprendre l'importance relative des différents éléments d'organisation et de préparation, dont les attentes reposent davantage sur des expériences passées mais non comprises que sur la connaissance du potentiel des interprètes dans un environnement donné, et qui ne distinguent pas la médiocrité des interprètes des mauvaises conditions d'interprétation - des opérations de sensibilisation pourraient assurer une meilleure adéquation entre les attentes et les possibilités.

Nous pensons notamment à des «mesures d'interfaçage» directes qui s'ajouteraient à la recherche et à la publication d'articles dans les revues spécialisées. Il serait envisageable par exemple de remettre systématiquement aux organisateurs un texte de sensibilisation d'une page environ à l'occasion de tout contact, voire de faire figurer ce texte dans les contrats d'engagement, et ce au même titre que les précisions sur les conditions générales de travail.

À une période où le marché de l'interprétation est en évolution et où des considérations pécuniaires conduisent certains organisateurs à des solutions de compromis dont ils ne réalisent pas toujours les implications, nous pensons qu'ils gagneront à être informés de manière à pouvoir prendre leurs décisions en sachant ce que peuvent leur apporter les interprètes en fonction des conditions dans lesquelles ils travaillent. 


\section{BIBLIOGRAPHIE}

DÉJEAN-LE-FÉAL, K. (1978): Lectures et improvisations - Incidences de la forme de l'énonciation sur la traduction simultanée, doctorat de $3^{\mathrm{e}}$ cycle, Université de la Sorbonne-Nouvelle, Paris.

GILE, D. (1983) : «Aspects méthodologiques de l'évaluation de la qualité du travail en interprétation simultanée», $M E T A, 28: 3$, septembre.

GILE, D. (1984a) : «Des difficultés de la transmission informationnelle en interprétation simultanée», Babel, $\mathrm{XXX}: 1$, janvier

GILE, D. (1984b) : «Les noms propres en interprétation simultanée», Multilingua, 3:2, juin.

GILE, D. (1985a): «Le modèle d'efforts et l'équilibre d'interprétation en interprétation simultanée», META, 30:1, mars.

GILE, D. (1985b) : «L'analyse dans la traduction humaine», actes de COGNITIVA 85, Paris, CESTA, vol. 1, pp. 63-67, juin.

HERBERT, J. (1952) : Manuel de l' interprète, Genève, Librairie de l'université Georg.

MOSER, B. (1978): «Simultaneous Interpretation: A Hypothetical Model and Its Practical Application», dans D. GERVER and H. Wallace SINAIKO, Language, Interpretation and Communication, NATO Conference Series, New York and London, Plenum Press.

QUICHERON, J.-B. (1984) : «Mieux interpréter aux congrès techniques, un défi inaccessible?», Lebende Sprachen, Nr. 1.

SELESKOVITCH, D. (1968) : l'Interprète dans les conférences internationales, Paris, Minard lettres modernes. 